\title{
Types of Diabetes that the Dipeptidyl Peptidase-4 Inhibitor May Act Effectively and Safely
}

\author{
Hidekatsu Yanai* and Hiroki Adachi \\ Department of Internal Medicine, National Center for Global Health and Medicine, Kohnodai Hospital, Chiba 272- \\ 8516, Japan
}

\begin{abstract}
Dipeptidyl peptidase-4 (DPP-4) inhibitors prevent the inactivation of glucagon-like peptide-1 (GLP-1). This protein, released from the gut following ingestion of a meal, stimulates insulin secretion and inhibits glucagon secretion. Compared with other anti-diabetic drugs, the pharmacological characteristics of DDP-4 inhibitor include improvement in postprandial hyperglycemia, low frequency of hypoglycemia, prevention of development of obesity, few adverse events. Taking account of pharmacological characteristic and our therapeutic experiences with DPP-4 inhibitor, we believe that DPP-4 inhibitor may be a useful and safe oral anti-diabetic drug for diabetes in the elderly people, diabetes complicated with obesity, chronic hepatitis/liver cirrhosis, and steroid-induced diabetes.
\end{abstract}

Keywords: chronic hepatitis, dipeptidyl peptidase-4, glucagon-like peptide-1, obesity, steroid-induced diabetes.

\section{INTRODUCTION}

The dipeptidyl peptidase-4 (DPP-4) inhibitor, which mainly prevents the inactivation of the glucagon-like peptide-1 (GLP-1), increases the endogenous GLP-1 concentrations $[1,2]$. GLP-1 is released from the intestine following meal ingestion and GLP-1 stimulates insulin secretion and inhibits glucagon secretion, which reduces plasma glucose levels $[1,3]$. Since GLP-1 is promptly inactivated by the enzyme, DPP-4, the half-life of GLP-1 is less than two minutes $[1,2]$. Increased insulin secretion and decreased glucagon secretion due to DDP-4 inhibition reduce hepatic glucose production [4-6]. DPP-4 inhibitor has beneficial effects on pancreatic $\beta$-cell structure and function [7]. Clinical trials using DPP-4 inhibitors show high tolerability and safety [2]. Several studies with a number of different DPP-4 inhibitors show that the number of adverse events is not increased in DPP-4 inhibitors-group compared to the placebo-groups [2]. Hypoglycemia is very rare (less than 3\%) during treatment with DPP-4 inhibitors as monotherapy or in combination with metformin or thiazolidinediones $[2,8-14]$. Low frequency of hypoglycemia may be explained by GLP-1-mediated glucose-dependent insulin secretion. Furthermore, many studies demonstrated that DPP-4 inhibitor do not increase body weight compared to thiazolidinediones, sulfonylurea and insulin $[2,10-16]$.

The pharmacological characteristics of DDP-4 inhibitors include 1) improvement in postprandial hyperglycemia, 2) low frequency of hypoglycemia, 3) prevention of development of obesity, 4) few adverse events. Here, we will introduce types of diabetes that DPP-4 inhibitor, sitagliptin, may act effectively and safely, by showing case reports.

*Address correspondence to this author at the Department of Internal Medicine, National Center for Global Health and Medicine, Kohnodai Hospital, Chiba 272-8516, Japan; Tel: +81-47-373-3501; Fax: +81-47-3721858; E-mail: dyanai@hospk.ncgm.go.jp

\section{MATERIALS, METHODS, AND RESULTS}

\section{Study Subject 1}

In May, 2010, a 74-year-old female patient was diagnosed as diabetes for the first time and referred to our department. Her fasting plasma glucose level was $113 \mathrm{mg} / \mathrm{dl}$ and hemoglobin A1c level was $8.2 \%$. Her body height, body weight and body mass index were $152.7 \mathrm{~cm}, 54.6 \mathrm{~kg}$ and $23.4 \mathrm{~kg} / \mathrm{m}^{2}$, respectively.

Before the use of sitagliptin, her postprandial blood glucose levels showed more than $150 \mathrm{mg} / \mathrm{dl}$ (Fig. 1). At 3 days after the start of sitagliptin, postprandial blood glucose levels and the area under the curve (AUC) of blood glucose levels were significantly decreased (Fig. 1). Interestingly, sitagliptin reduced serum insulin levels at 14:00, 18:00, and 20:00, and the AUC of serum insulin levels (Fig. 2).

\section{Study Subject 2}

A 46-year-old obese diabetic man with mental retardation who cannot control appetite has been treated as type 2 diabetes mellitus for previous 5 years. Metformin (1500 $\mathrm{mg}$ /day), pioglitazone (15 mg/day), miglitol (150 mg/day), and mitiglinide $(60 \mathrm{mg} / \mathrm{day})$ were used to treat diabetes. Since he could not perform a dietary therapy due to mental retardation, he is constantly in and out of hospital. On April 19, 2010, his blood glucose level was $382 \mathrm{mg} / \mathrm{dl}$, and his hemoglobin A1c was $10.7 \%$. His body height, body weight and body mass index were $170 \mathrm{~cm}, 90 \mathrm{~kg}$ and $31 \mathrm{~kg} / \mathrm{m}^{2}$, respectively. He was again admitted to our hospital for treatment of diabetes.

The patient could not keep to a dietary therapy (1600 $\mathrm{kcal} /$ day), and was a frequent repeat offender consuming additional calories daily. When discovered we stopped mitiglinide and started intensive insulin therapy (Fig. 3). 30 days post admission we discontinued miglitol and insulin and initiated treatment with sitagliptin. Since his blood glucose levels were significantly ameliorated (Fig. 3), he left 
A

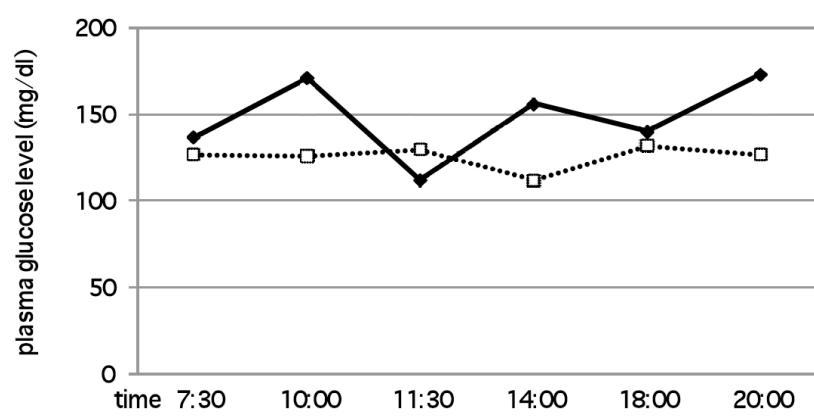

B

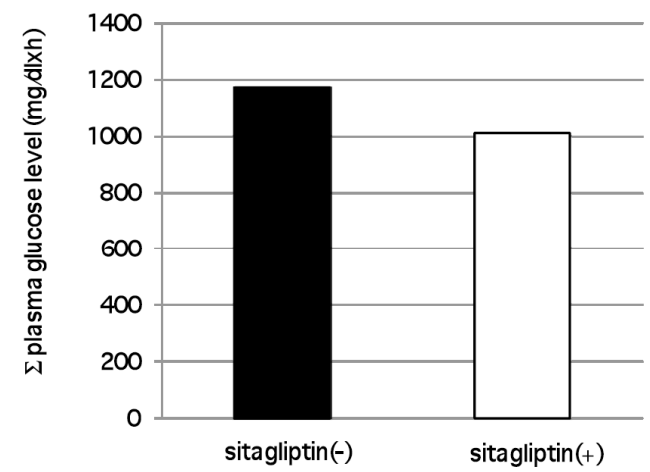

Fig. (1). (A) Changes of plasma glucose levels. Closed and opened boxes indicate values before and after the use of sitagliptin (50 $\mathrm{mg} /$ day). 7:30, 10:00, 11:30, 14:00, 18:00, and 20:00 mean before breakfast, 2 hours after breakfast, before lunch, 2 hours after lunch, before dinner, and 2 hours after dinner, respectively. (B) The area under the curve of plasma glucose levels of before (closed bar) and after (opened bar) the use of sitagliptin ( $50 \mathrm{mg}$ /day).

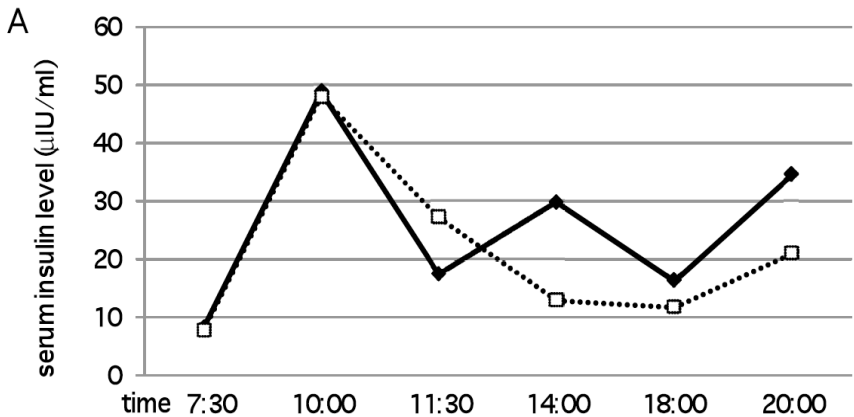

B

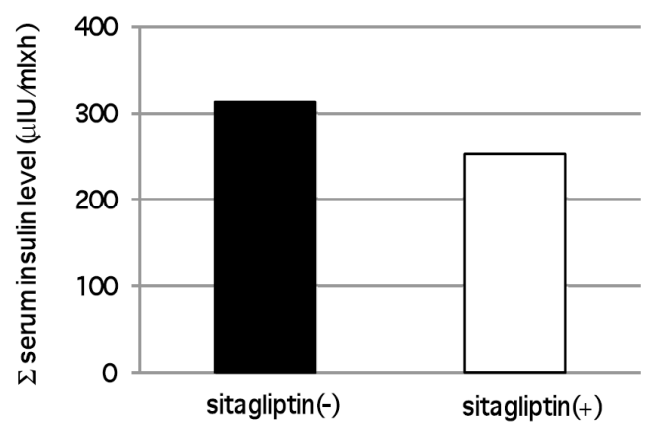

Fig. (2). (A) Changes of serum insulin levels. Closed and opened boxes indicate values before and after the use of sitagliptin (50 $\mathrm{mg}$ /day). 7:30, 10:00, 11:30, 14:00, 18:00, and 20:00 mean before breakfast, 2 hours after breakfast, before lunch, 2 hours after lunch, before dinner, and 2 hours after dinner, respectively. (B) The area under the curve of serum insulin levels of before (closed bar) and after (opened bar) the use of sitagliptin $(50 \mathrm{mg} /$ day).

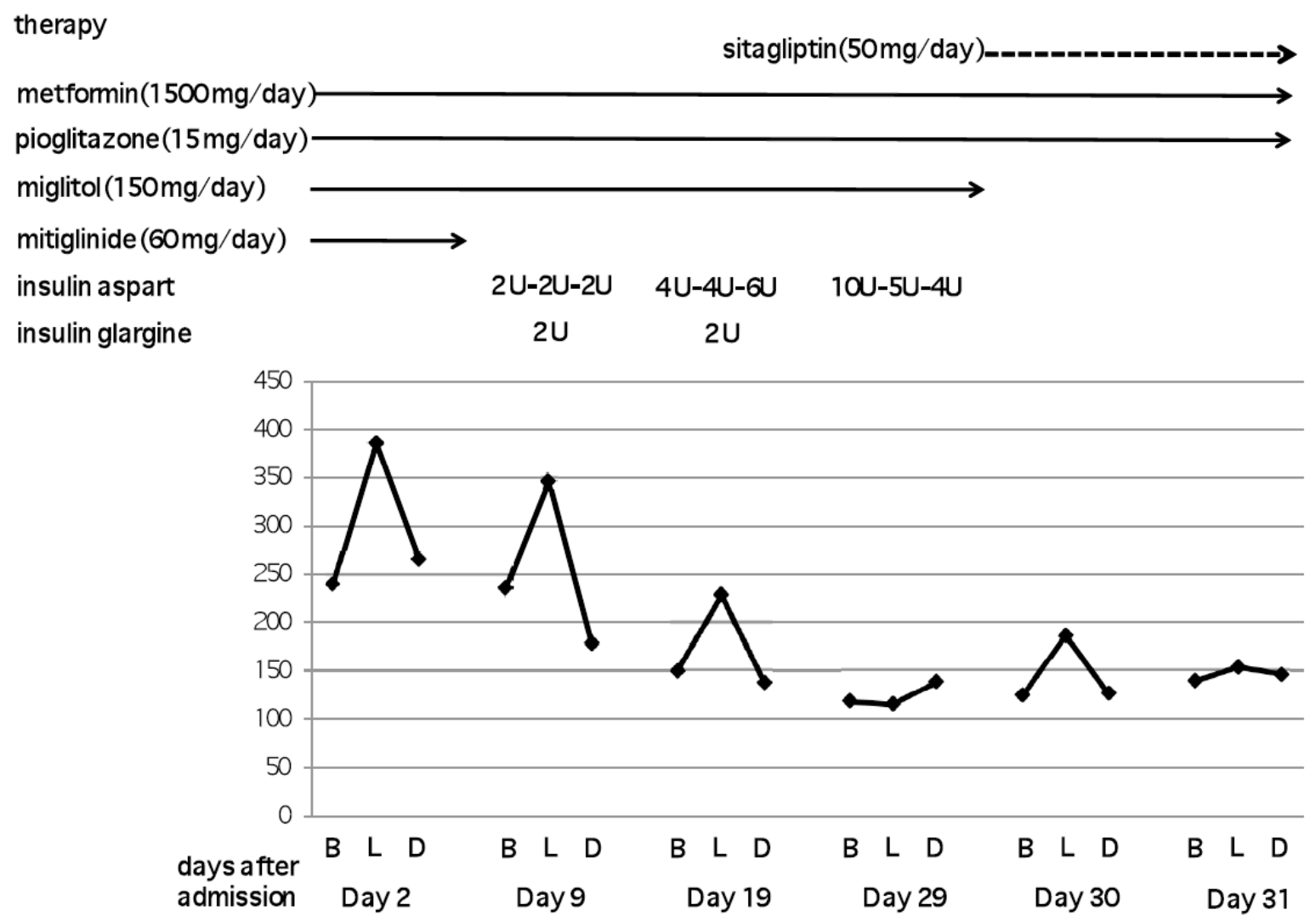

Fig. (3). Changes of blood glucose levels. B, L, and D mean before breakfast (7:30), before lunch (11:30), and before dinner (18:00), respectively. 
the hospital on May 20, 2010 (Day 32). After discharge, hemoglobin A1c level was reduced $(9.1 \%$ on June 1,2010$)$, and his body weight has been decreasing (Fig. 4).

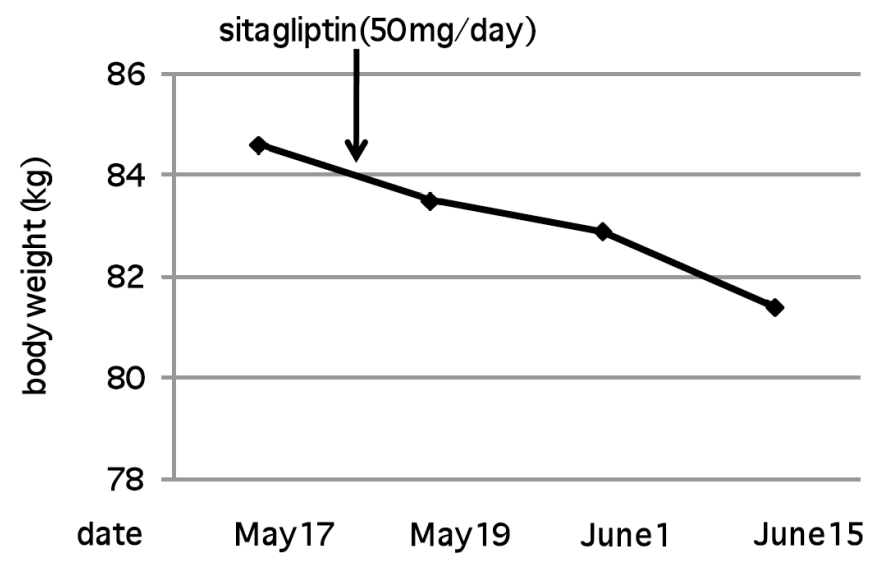

Fig. (4). Changes of body weight.

\section{Study Subject 3}

In March, 2010, a 56-year-old woman has been found to show remarkable hyperglycemia (plasma glucose level, 712 $\mathrm{mg} / \mathrm{dl}$; hemoglobin A1c level, 11.4\%). Her body height, body weight and body mass index were $150.4 \mathrm{~cm}, 42.9 \mathrm{~kg}$ and $19.0 \mathrm{~kg} / \mathrm{m}^{2}$, respectively. Elevated serum aspartate transaminase (AST, $75 \mathrm{IU} / 1$, normal range; 10-35 IU/1) and alanine transaminase (ALT, $64 \mathrm{IU} / 1$, normal range; 5-40 IU/l) levels were observed. The high titer of anti-hepatitis C virus (HCV) antibody (61.1) and high quantity of RNA for HCV genotype $1 b$ (7.3). Serum total bilirubin level was within normal range $(0.6 \mathrm{mg} / \mathrm{dl}$, normal range; 0.3-1.2 $\mathrm{mg} / \mathrm{dl})$, and slight abnormal levels of serum albumin (3.5 $\mathrm{g} / \mathrm{dl}$, normal range; 3.9-5.0 g/dl), platelet $\left(9.8 \times 10^{4} / \mu \mathrm{l}\right.$, normal range; $\left.15.0-35.0 \times 10^{4} / \mu 1\right)$, and prothorombin time (13.3 seconds, normal range; 10.0-13.0 seconds) were observed. Based on these laboratory data and ultrasound of her liver, she has been diagnosed as having not liver cirrhosis but chronic active hepatitis.

When her blood glucose levels were decreased by the intensive insulin therapy, we started to use glimepiride (1 $\mathrm{mg}$ /day) and metformin (750 mg/day). This combination of sulfonylurea and biguanides was effective to reduce her plasma glucose levels (blood glucose levels, $120-187 \mathrm{mg} / \mathrm{dl}$ ), however, this combination therapy deteriorated liver function (AST, from 60 to $135 \mathrm{IU} / \mathrm{l}$; ALT, from 48 to 84 IU/l), and then, we changed from this combination therapy to the DPP-4 inhibitor, sitagliptin $(50 \mathrm{mg} / \mathrm{day})$. The hypoglycemic ability of sitagliptin was comparable to the ability of the combination of sulfonyurea and biguanides (blood glucose levels one week after the start of sitagliptin, $101-150 \mathrm{mg} / \mathrm{dl}$ ), and sitagliptin ameliorated liver function (AST, from 135 to $54 \mathrm{IU} / 1$; ALT, from 84 to $64 \mathrm{IU} / 1$ ) [17].

\section{Study Subject 4}

An 81-year-old female patient with polymyalgia rheumatica was treated with daily $20 \mathrm{mg}$ prednisolone, and she developed steroid-induced diabetes. Her hemoglobin A1c level was $8.3 \%$, and daily $30-40$ unites of insulin were needed to treat hyperglycemia. Her fasting blood glucose levels were relatively low (blood glucose levels, 88-146 $\mathrm{mg} / \mathrm{dl}$ ), and postprandial glucose levels were remarkably elevated (blood glucose levels, 200-417 mg/dl). Since she refused to use insulin at home, we started to use metformin $(250 \mathrm{mg} /$ day $)$, nateglinide $(270 \mathrm{mg} /$ day $)$, and pioglitazone $(15 \mathrm{mg} /$ day). Since 6-8 unites of insulin were needed to reduce blood glucose levels below $200 \mathrm{mg} / \mathrm{dl}$, we changed from nateglinide to sitagliptin $(50 \mathrm{mg} / \mathrm{dl})$. After the change of therapy, her postprandial glucose levels were significantly decreased (blood glucose levels, 99-168 mg/dl) and finally the addition of insulin was not required [18]. After discharge, hemoglobin A1c level has been decreasing (Fig. 5).

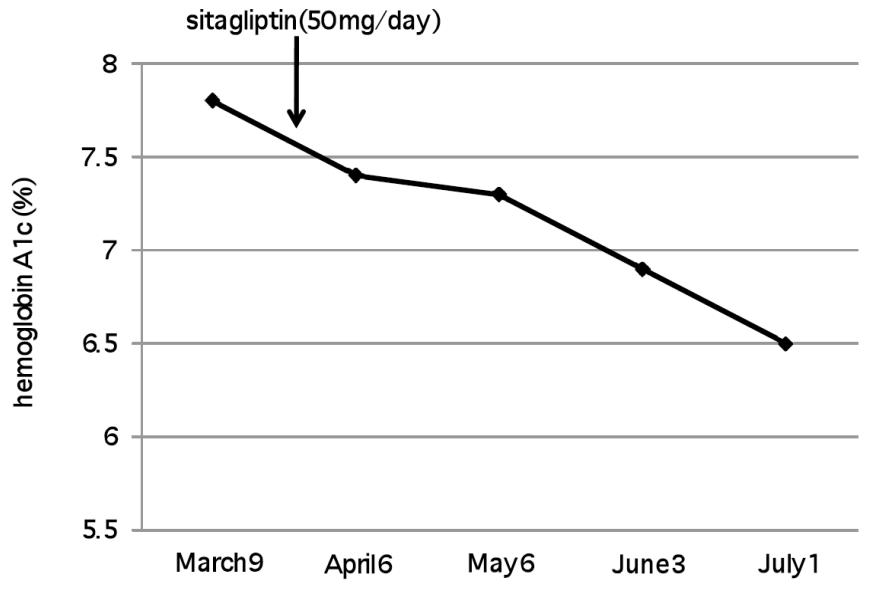

Fig. (5). Changes of hemoglobin A1c levels.

\section{DISCUSSION}

Sitagliptin reduced postprandial blood glucose levels and the AUC of blood glucose levels, in spite of decreased AUC of serum insulin levels in study subject 1, suggesting that sitagliptin ameliorates insulin resistance. Hypoglycemia was not observed at every time point. This observation suggests that DPP-4 inhibitor improves insulin sensitivity even in the elderly people and is also safe and efficacious drug for diabetes in the elderly people. However, in general, elderly people have renal dysfunction due to declined glomerular filtration rate (GFR) with aging. Plasma levels of sitagliptin are increased by 2 -fold in patients with moderate renal dysfunction (creatinin clearance $30-50 \mathrm{~mL} / \mathrm{min}$ ) and by $4-$ fold in patients with severe renal dysfunction (creatinin clearance $<30 \mathrm{~mL} / \mathrm{min}$ ) [19]. Increased circulating levels of sitagliptin induce severe hypoglycemia. Making dose adjustment is recommended for these patients.

Sitagliptin improved glucose metabolism and decreased body weight in obese diabetic patient (study subject 2) who could not perform a dietary therapy due to mental retardation, suggesting the usefulness of DPP-4 inhibitors for diabetes complicated with obesity. Insulin resistance is commonly observed in obese people. Sitagliptin-mediated amelioration in insulin resistance found in study subjects 1 may support the effectiveness of DPP-4 inhibitors for the glycemic control in obese people. Several studies also demonstrated that DPP-4 inhibitor do not increase body weight compared to thiazolidinediones, sulfonylurea and insulin $[2,10-16]$.

Although a high prevalence of glucose intolerance in patients with chronic hepatitis has been reported [20], few studies have evaluated what is the most effective and safe therapy for diabetes mellitus in chronic hepatitis patients. 
We experienced a diabetic 56-year-old female patient complicated with chronic hepatitis C (study subject 3 ), who could be effectively and safely treated by the DPP-4 inhibitor, sitagliptin [17].

The treatment of diabetes complicated with chronic hepatitis/liver cirrhosis is difficult, because most of the oral anti-diabetic drugs (sulfonyurea, biguanides, and thiazolidines) are metabolized in the liver and patients frequently show hypoglycemia. Relatively low fasting plasma glucose levels due to insufficient hepatic gluconeogenesis, and postprandial hyperglycemia due to insufficient hepatic glucose uptake are characteristics for diabetes complicated with chronic hepatitis/liver cirrhosis. Since DPP-4 inhibitor induces GLP-1-mediated meal ingestion (glucose)-dependent insulin secretion, DPP-4 inhibitor improves postprandial glucose levels but do not cause hypoglycemia. Further, since sitagliptin is minimally metabolized and over $80 \%$ of the compound is excreted unchanged in the urine, hepatic insufficiency do not seem to alter the pharmacokinetics of sitagliptin [21]. DPP-4 inhibitors may be effective and safe drug for diabetes complicated with chronic hepatitis/liver cirrhosis. Currently, another DPP-4 inhibitor, vildagliptin, is available for the treatment of type 2 diabetes. Based on observations using higher doses than ones proposed for clinical use, a caution for liver dysfunction was built in to the label [2]. Although moderate liver dysfunction has been reported to have no clinically meaningful effect on the pharmacokinetics of sitagliptin [22], liver function tests should be performed prior to initiation of use of DPP-4 inhibitors, and periodically thereafter.

The mechanisms for steroid-induced diabetes may include increased hepatic glucose output and insulin resistance. The characteristics for steroid-induced diabetes are normal fasting plasma glucose levels and postprandial hyperglycemia [23]. We experienced a patient with steroidinduced diabetes, whose blood glucose levels were ameliorated by the use of sitagliptin [18]. The DPP-4 inhibitor improves postprandial hyperglycemia and insulin resistance, and rarely induces hypoglycemia. Therefore, DPP-4 inhibitor may be a useful and safe oral anti-diabetic drug for steroid-induced diabetes.

The incidence of gastrointestinal-related adverse experiences during treatments with sitagliptin has been reported to be approximately $10 \%$ [24]. None of our patients studied complained of gastrointestinal-related adverse experiences. Further, none of our patients showed laboratory adverse experiences including liver dysfunction and renal dysfunction.

\section{CONCLUSION}

The pharmacological characteristics of DDP-4 inhibitor includes improvement in postprandial hyperglycemia, low frequency of hypoglycemia, prevention of development of obesity, few adverse events. DPP-4 inhibitor may be an effective and safe oral anti-diabetic drug for diabetes in the elderly, diabetes complicated with obesity, chronic hepatitis/liver cirrhosis, and steroid-induced diabetes (Table 1).
Table 1. Types of Diabetes that DPP4 Inhibitor May Act Effectively and Safely
1) Elderly people
2) Obesity
3) Chronic hepatitis, liver cirrhosis
4) Steroid induced diabetes

\section{ACKNOWLEDGEMENT}

This study was supported by the Grant of National Center for Global Health and Medicine, Japan.

\section{REFERENCES}

[1] Drucker DJ, Nauck MA. The incretin system: glucagon-like peptide-1 receptor agonists and dipeptidyl peptidase- 4 inhibitors in type 2 diabetes. Lancet 2006; 368: 1696-705.

[2] Ahrén B. Use of DPP-4 inhibitors for type 2 diabetes: focus on sitagliptin. Diabetes Metab Syndr Obes: Targets Therapy 2010; 3: 31-41.

[3] Ahrén B. Glucagon-like peptide-1 (GLP-1): a gut hormone of potential interest in The treatment of diabetes. Bioessays 1998; 20: 642-51.

[4] Ahrén B, Landin-Olsson M, Jansson PA, et al. Inhibition of dipeptidyl peptidase-4 reduces glycemia, sustains insulin levels and reduces glucagon levels in type 2 diabetes. J Clin Endocrinol Metab 2004; 89: 2078-84.

[5] Ahrén B, Pacini G, Foley JE, Schweizer A. Improved meal-related beta-cell function and insulin sensitivity by the dipeptidyl peptidase-IV inhibitor vildagliptin in metformin-treated patients with type 2 diabetes over 1 year. Diabetes Care 2005; 28: 1936-40.

[6] Balas B, Baig MR, Watson C, et al. The dipeptidyl peptidase IV inhibitor vildagliptin suppresses endogenous glucose production and enhances islet function after single dose administration in type 2 diabetic patients. J Clin Endocrinol Metab 2007; 92: 1249-55.

[7] Cheng Q, Law PK, de Gasparo M, Leung PS. Combination of the dipeptidyl peptidase IV inhibitor LAF237 [(S)-1-[(3-hydroxy-1adamantyl)ammo]acetyl-2-cyanopyrrolidine] with the angiotensin II type 1 receptor antagonist valsartan [N-(1-oxopentyl)-N-[[2'(1H-tetrazol-5-yl)-[1,1'-biphenyl]-4-yl]methyl]-L-valine] enhances pancreatic islet morphology and function in a mouse model of type 2 diabetes. J Pharmacol Exp Ther 2008; 327: 683-91.

[8] Nonaka K, Kakikawa T, Sato A, et al. Efficacy and safety of sitagliptin monotherapy in Japanese patients with type 2 diabetes. Diabet Res Clin Pract 2008; 79: 291-8.

[9] Kashiwagi A, Tajima N, Kadowaki T, et al. Sitagliptin added to ongoing treatment with pioglitazone improved glycemic control and was well tolerated in Japanese patients with type 2 diabetes. Diabetes 2008; (Suppl 1): A590.

[10] Aschner P, Kipnes MS, Lunceford JK, Sanchez M, Mickel C, Williams-Herman DE. Effect of the dipeptidyl peptidase-4 inhibitor sitagliptin as monotherapy on glycemic control in patients with type 2 diabetes. Diabetes Care 2006; 29: 2632-7.

[11] Raz I, Hanefeld M, Xu L, Caria C, Williams-Herman D, Khatami H. Efficacy and safety of the dipeptidyl peptidase-4 inhibitor sitagliptin as monotherapy in patients with type 2 diabetes mellitus. Diabetologia 2006; 49: 2564-7.

[12] Goldstein BJ, Feinglos MN, Lunceford JK, Johnson J, WilliamsHerman DE. Effect of initial combination therapy with sitagliptin, a dipeptidyl peptidase-4 inhibitor, and metformin on glycemic control in patients with type 2 diabetes. Diabetes Care 2007; 30: 1979-87.

[13] Charbonnel B, Karasik A, Liu J, Wu M, Meininger G. Efficacy and safety of the dipeptidyl peptidase-4 inhibitor sitagliptin added to ongoing metformin therapy in patients with type 2 diabetes inadequately controlled with metformin alone. Diabetes Care 2006; 29: $2638-43$.

[14] Scott R, Wu L, Sanchez M, Stein P. Efficacy and tolerability of the dipeptidyl peptidase-4 inhibitor sitagliptin as monotherapy over 12 weeks in patients with type 2 diabetes. Int J Clin Pract 2007; 61: 171-80. 
[15] Hermansen K, Kipnes M, Luo E, Fanurik D, Khatami H, Stein P. Efficacy and safety of the dipeptidyl peptidase-4 inhibitor, sitagliptin, in patients with type 2 diabetes mellitus inadequately controlled on glimepiride alone or on glimepiride and metformin. Diabet Obes Metab 2007; 9: 733-45.

[16] Rosenstock J, Brazg RG, Andryuk PJ, Lu K, Stein P. Efficacy and safety of the dipeptidyl peptidase-4 inhibitor sitagliptin added to ongoing pioglitazone therapy in patients with type 2 diabetes: a 24week, multicenter, randomized, double-blind, placebo-controlled, parallel-group study. Clin Ther 2006; 28: 1556-68.

[17] Yanai H. The DPP-4 inhibitor, sitagliptin, for the treatment of diabetes complicated with chronic hepatitis C. Hepatobiliary Pancreat Dis Int 2010; 9: 442-3.

[18] Yanai H, Masui Y, Yoshikawa R, Kunimatsu J, Kaneko H. Dipeptidyl peptidase-4 inhibitor for steroid-induced diabetes. World J Diabetes 2010; 1: 99-100.

[19] Bergman AJ, Stevens C, Zhou Y, et al. Pharmacokinetic and pharmacodynamic properties of multiple oral doses of sitagliptin, a dipeptidyl peptidase-IV inhibitor: a double-blind, randomized, placebo-controlled study in healthy male volunteers. Clin Ther 2006; 28: 55-72.

[20] Chen LK, Hwang SJ, Tsai ST, Luo JC, Lee SD, Chang FY. Glucose intolerance in Chinese patients with chronic hepatitis C. World J Gastroenterol 2003; 9: 505-8.

[21] Vincent SH, Reed JR, Bergman AJ, et al. Metabolism and excretion of the dipeptidyl peptidase 4 inhibitor [14C]sitagliptin in humans. Drug Metab Dispos 2007; 35: 533-8.

[22] Migoya EM, Stevens CH, Bergman AJ, et al. Effect of moderate hepatic insufficiency on the pharmacokinetics of sitagliptin. Can J Clin Pharmacol 2009; 16: 165-70.

[23] Iwamoto T, Kagawa Y, Naito Y, Kuzuhara S, Kojima M. Steroidinduced diabetes mellitus and related risk factors in patients with neurologic diseases. Pharmacotherapy 2004; 24: 508-14.

[24] Aschner P, Katzeff HL, Guo H, et al, Sitagliptin Study 049 Group. Efficacy and safety of monotherapy of sitagliptin compared with metformin in patients with type 2 diabetes. Diabetes Obes Metab 2010; 12: 252-61.

(C) Yanai and Adachi; Licensee Bentham Open.

This is an open access article licensed under the terms of the Creative Commons Attribution Non-Commercial License (http:/creativecommons.org/licenses/by-nc/3.0/) which permits unrestricted, non-commercial use, distribution and reproduction in any medium, provided the work is properly cited. 